\title{
Effect of pregnancy-lactation overlap on the current pregnancy outcome in women At Assiut governorate
}

\author{
Azza Salem Abd El-Rhman, Omar Mamdouh Shaaban \& Hamida Alam El -dien Abdel Hafize. \\ Nursing specialist, Central hospital of El Ganaiem, \\ Assistant Professor of Obstetrics \& Gynecology, Faculty of Medicine. AssiutUniversity, \\ Lecturer of obstetrics \& Gynecological Nursing. Faculty of Nursing. Assiut University.
}

\begin{abstract}
Background: Pregnancy during breastfeeding is common in Egypt and is often unplanned, Overlap between pregnancy and breastfeeding was more common for short birth intervals and it could increase the risk of mothers and prenatal adverse outcomes. Aim of the study: was to study the effect of lactation during pregnancy on the maternal and prenatal outcome. Subjects and Methods: Prospective- cohort study, 540 women

convenient sample:conducted in six maternal and child health centers in Assiut governorate (Kolta, Hay ktharb, Elarbaeen, Hay shark, El Ganaiem and Manfalote MCH). Women were divided into two groups (270) Women who got pregnant during breastfeeding and (270) women who got pregnant after weaning. Results: The miscarriage rate was not statistically significant $(\mathrm{P}=0.284)$.however, pregnancy during breastfeeding women had higher incidence of Anima (54.1\%), IUGR (16.7\%), CS (43.7\%), Prolonged labor (13.3\%) and low birth weights (LBW) (14.4\%) compared with pregnant after weaning women . The results of present study revealed that there was no significant relation between the duration of lactation during the current pregnancy and complications.

Conclusion: breastfeeding during pregnancy doesn't increase the miscarriage rate. However, increases the risk of IUGR, anemia, prolonged labor, LBW and CS. Recommendations: Women should be better educated about the immediate interpregnancy interval and nutrition.
\end{abstract}

\section{Keywords: lactation-pregnancy overlap, LAM \& adverse pregnancy outcomes}

\section{Introduction}

Pregnancy during breastfeeding is common in Egypt and is often unintended. There is great potential for using lactation as a method of contraception in this community. Lactational amenorrhea method (LAM) has been integrated in the family planning program of many developing countries including Egypt. More than $95 \%$ of women in rural Egypt breastfed the child before their current pregnancy; $25.3 \%$ conceived while breastfeeding. Conception occurred during the first 6 months postpartum in $4.4 \%$, before resumption of menstruation in $15.1 \%$ and while exclusively or almost exclusively breastfeeding in $28.1 \%$. Only 10 pregnancies $(1.5 \%)$ occurred when all the prerequisites of the lactational amenorrhea method of contraception (LAM) were present. $29 \%$ of pregnancies conceived during breastfeeding were unintended, $10 \%$ of women had considered terminating their pregnancy while $4.4 \%$ of them reported trying to do so. However, LAM use is limited to six months because infant-feeding guidelines recommend that supplementation with other foods should begin at six months after delivery (Shaaban et al., 2008 \& Khella et al., 2004).

The use of LAM depends on 3 essential criteria; (1) amenorrhea, which is defined as the absence of 2 consecutive days of bleeding or spotting after postpartum day number 56 (or 2 months); (2) fully or nearly fully breastfeeding, which is defined practically as breastfeeding the infant at least every 4 hours during the day and at least every 6 hours during the night, and not feeding the infant other foods or liquids that replace breastfeeds; (3) being fewer than 6 months post partum. When LAM criteria are met, the pregnancy rate is less than $2 \%$ during the first 6 months (Speroff et al., 2008).

Lactation generally extends beyond the period of LAM efficacy and its prerequisites usually expire unexpectedly. Reluctance to use of a long-term method of contraception during lactation may result in unplanned pregnancy, and sometimes unwanted childbirth. Previous study in the same setting showed that one in four women conceived while breastfeeding, and nearly one in three of these pregnancies were unplanned. in rural Egypt, as many as one-third of all pregnancies are reported to be unintended, $10 \%$ of pregnancies are sufficiently unwanted to lead mothers to consider having an induced abortion; almost 5\% of couples reported taking steps to do so. In Egypt, it is estimated that, each year, 216000 women are admitted to hospital with complications of abortion, despite abortion being legal (Shaaban et al., 2008).

Birth spacing is mandatory to make pregnancy safer, and so the WHO endorses a minimum birth-to- 
pregnancy interval of two years to reduce the incidence of maternal and fetal risks in each pregnancy (Tilley et al., 2009). Several studies have shown that women with a short inter- pregnancy interval less than 24 months increases the risk of adverse prenatal outcomes such as; abortion, preterm birth (less than 37-week of gestation), low birth weight (less than $2500 \mathrm{~g}$ at birth), intrauterine growth retardation (IUGR) and fetal death. Moreover, women with shorter inter-pregnancy intervals have a higher risk of maternal mortality, hypertensive disorders of pregnancy, peripartal bleeding and anemia (Albadran, et al, 2013).

Maternal nutritional status is considered an important risk factor for the progression of labor and birth weight. Poor birth outcomes, such as experiencing a prolonged labor or being small for gestational age, represent a risk factor for maternal as well as infant morbidity and mortality, Nutrition deserves special attention during pregnancy and breastfeeding because of the high nutrient needs and the critical role of appropriate nutrition for the fetus and infant (Ministry of Health, 2006).

Overlap between pregnancy and breastfeeding was more common for short birth intervals, when births are closely spaced, overlap of breastfeeding with pregnancy is more prevalent, which could affect the breastfeeding of the newborn. Consequently, short intervals could indirectly increase the risk of adverse neonatal/infant outcomes through changes in breastfeeding patterns or the composition and/or quantity of breast milk secondary to breastfeedingpregnancy overlap (Pareja, 2007).

It has been suggested that this overlap could produce suboptimal outcomes for both pregnancy and subsequent lactation, such as IUGR of the fetus, risk of depletion of the nutrient stores in the mother, alteration of the breastfeeding patterns, and a reduction of milk for the breastfed child Sengul et al., (2012) Widespread beliefs hold that pregnancy and lactation are incompatible and that breast-feeding during pregnancy is harmful to the fetus, For example, breast milk sometimes is viewed as harmful to the fetus; conversely, pregnancy sometimes is thought to spoil or damage the milk. The fetus, the toddler, or even the mother may be viewed as being at risk if pregnancy and lactation overlap Madarshahian, (2012)

Women in our culture having a high total fertility rate and almost inadequate dietary intake that should necessary need a appropriate interval between breastfeeding and the next pregnancy. Therefore, it is of great health importance to know the effects of pregnancy during lactation on pregnancy outcome (Shaaban et al., 2008).

\section{Aim of the study}

1. Study the effect of pregnancy during lactation on the maternal and prenatal outcome.

2. Study the effect of duration of lactation on the pregnancy outcome.

3. Study the effect of the time of weaning of the last child on the pregnancy outcome.

\section{Study hypothesis}

1. Is there is an effect of pregnancy during lactation on the maternal and prenatal outcome?

2. Is the pregnancy harmful to breast feeding baby?

3. What is the effect of duration of lactation on the pregnancy outcome?

4. What is the effect of the time of weaning of the last child on the pregnancy outcome?

Subject and methods

Research design: Prospective- cohort study carried out in this study.

Setting

The study was carried out in antenatal and maternity units of six maternal and child health centers $(\mathrm{MCH})$ in Assiut governorate (Kolta $\mathrm{MCH}$, Hay ktharb $\mathrm{MCH}$, Elarbaeen $\mathrm{MCH}$, Hay shark $\mathrm{MCH}$, El Ganaiem MCH and Manfalote MCH).

Study population

The study had included all multiparous pregnant women presented to any of the above recruitment $\mathrm{MCH}$ centers in the first trimester of pregnancy (less than 12 weeks pregnant). However, women with history of abortion in the last pregnancy or history of any medical complication had been excluded from participation. Additionally, women who did not breastfeed their last child and those who were living in districts away from recruitment centers or refused to participate in the study had also been excluded.

A sample size of 540 women convenient sample in two groups (270 in each group) had been estimated based on the primary outcome (abortion rate in both groups). the general miscarriage rate in the six conducted maternal and child health centers $(\mathrm{MCH})$ in years 2011 is about $15 \%$ of total pregnant women attending to this six centers based on Directorate of Health reports in Assuit .Using two sided chi-square (X2) test with $\alpha$ of 0.05 , a total sample size of at least) will have $95 \%$ power to detect a $10 \%$ difference in the abortion rate in both groups fodds ratio of 1.89 \} assuming a lost to follow up rate of $10 \%$ (Epi-info TM, CDC, USA).

\section{Intervention}

The study protocol had been first approved from the Institutional review board and the Ministry of Health approval of collecting data from their affiliated Centers was also obtained. 


\section{Tools}

Tool (1) A structured interviewing sheet, it was structured to include:

The questions included those related to personal (included contact details), obstetrical, breastfeeding histories and family planning history. Moreover, it included questions regarding pregnancy intention and dietary intake of calcium.

The Interviews were conducted in a private room and started by greeting from the investigator side followed by description of the study and assessing of the patients legibility and willingness to participate. The non-interventional nature of the stud and respect of patient's confidentiality were keeping clear to the patient and their oral consent to participate had been obtained.

Tool (2) Follow up sheet: It was designed to assess Information about the effect of breastfeedingpregnancy overlaps on pregnancy outcome and in labor and newborn outcome.

The researcher trained at least two nurses in each recruitment center (six training days) about who to complete the data sheet during the routine follow-up visits of the pregnant mothers. Missing data were obtained by phone calls to the pregnant mother by the investigators.

The researcher met the women for four time until delivery and follow-up them by telephone between visits:

At the first trimester when we met the women for the first time.

The $1^{\text {st }}$ Follow-up12 - 24 weeks of pregnancy (second trimester), usually at the time of tetanus vaccination.

The $2^{\text {nd }}$ Follow-up $24-36$ weeks of pregnancy (second trimester).

The $3^{\text {rd }}$ Follow-up At the time of delivery or after that Woman in both groups were followed up during their routine antenatal visits to the centers until delivery to know if there were any complication occurred during the current pregnancy (miscarriage, vaginal bleeding during pregnancy, placental separation (partially or completely), and delayed fetal growth, elevation of blood sugar, elevation of blood pressure, eclampsia and anemia. Moreover, participants were followed up during delivery to report the mode of delivery, the time of labor, any intrapartum or postpartum complication (ante-partum or post-partum hemorrhage, premature rupture of membranes and prolonged labor). In those who were delivered by cesarean section, the CS indications were also reported. The condition of the newborn, his or her weight and referral to $\mathrm{PCO}$ and the indication of that were also reported.

Every woman in both groups was followed up until delivery to complete the required data. Before ending the meeting, the researcher gave the participants a follow up card which included: Patient serial number, Basic information about each case as name, address, Follow up schedule. And Information about the nutrition and warning signs during pregnancy.

The researcher follow-up the women and the baby at the time of delivery, for the women who couldn't reach to them at the time of labor, the researcher follow-up those women by telephone and by the hospital record.

\section{Statistical analysis}

The collected data was coded, tabulated and analyzed using the statistical package for social science programs (SPSS) version 16 windows Microsoft .Continuous data was expressed as frequency, percentage, means and SD .Discrete data was expressed as frequency and percentage. Comparison between variables was made using probability ( $\mathrm{P}$ value), less than 0.05 was considered significant and less than 0.001 was considered highly significant. 
Results

Table (1): Demographic characteristics of the study participants.

\begin{tabular}{|c|c|c|c|c|c|}
\hline \multirow{2}{*}{ Demographic Characteristics } & \multicolumn{2}{|c|}{ PDBF $(n=270)$} & \multicolumn{2}{|c|}{ PAW $(n=270)$} & \multirow{2}{*}{ P-value } \\
\hline & No. & $\%$ & No. & $\%$ & \\
\hline \multicolumn{5}{|l|}{ Age } & \multirow{5}{*}{$0.000 *$} \\
\hline$<25$ years & 71 & 26.3 & 35 & 13.0 & \\
\hline $25-<30$ years & 92 & 34.0 & 76 & 28.1 & \\
\hline $30-<35$ years & 52 & 19.3 & 85 & 31.5 & \\
\hline$\geq 35$ years & 55 & 20.4 & 74 & 27.4 & \\
\hline Mean \pm SD & \multicolumn{2}{|c|}{$28.82 \pm 6.18$} & \multicolumn{2}{|c|}{$30.52 \pm 5.20$} & $0.001 *$ \\
\hline \multicolumn{5}{|l|}{ Husband occupation } & \multirow{3}{*}{$0.033 *$} \\
\hline Working near home & 235 & 87.0 & 250 & 92.6 & \\
\hline Working abroad & 35 & 13.0 & 20 & 7.4 & \\
\hline \multicolumn{5}{|l|}{ Level of education } & \multirow{3}{*}{0.649} \\
\hline Illiterate or Read \& write & 93 & 34.4 & 88 & 32.6 & \\
\hline Educated & 177 & 65.6 & 182 & 67.4 & \\
\hline \multicolumn{5}{|l|}{ Occupation } & \multirow{3}{*}{0.643} \\
\hline Working & 87 & 32.2 & 82 & 30.4 & \\
\hline Not working & 183 & 67.8 & 188 & 69.6 & \\
\hline
\end{tabular}

$(P D B F)$ pregnancy during breastfeeding

(*) Statistically significant difference

(PAW) pregnancy after weaning

Table (2): Obstetric history in the two study groups.

\begin{tabular}{|c|c|c|c|c|c|}
\hline \multirow{2}{*}{ Items } & \multicolumn{2}{|c|}{ PDBF $(n=270)$} & \multicolumn{2}{|c|}{$\operatorname{PAW}(n=270)$} & \multirow{2}{*}{ P-value } \\
\hline & No. & $\%$ & No. & $\%$ & \\
\hline \multicolumn{5}{|l|}{ Duration of marriage } & \multirow{3}{*}{$0.000 *$} \\
\hline$<10$ years & 197 & 73.0 & 141 & 52.2 & \\
\hline$\geq 10$ years & 73 & 27.0 & 129 & 47.8 & \\
\hline Mean \pm SD & \multicolumn{2}{|c|}{$7.47 \pm 5.37$} & \multicolumn{2}{|c|}{$9.34 \pm 4.53$} & $0.000 *$ \\
\hline \multicolumn{5}{|l|}{ previous deliveries } & \multirow{3}{*}{$0.000^{*}$} \\
\hline One & 104 & 38.5 & 42 & 15.6 & \\
\hline$>1$ & 166 & 61.5 & 228 & 84.4 & \\
\hline Mean \pm SD & \multicolumn{2}{|c|}{$2.39 \pm 1.54$} & \multicolumn{2}{|c|}{$2.64 \pm 1.18$} & $0.036^{*}$ \\
\hline \multicolumn{5}{|l|}{ living children } & \multirow{5}{*}{$0.000 *$} \\
\hline One & 110 & 40.7 & 43 & 15.9 & \\
\hline Two & 71 & 26.4 & 100 & 37.1 & \\
\hline Three & 39 & 14.4 & 91 & 33.7 & \\
\hline Four of more & 50 & 18.5 & 36 & 13.3 & \\
\hline Mean \pm SD & \multicolumn{2}{|c|}{$2.26 \pm 1.44$} & \multicolumn{2}{|c|}{$2.49 \pm 1.02$} & $0.031 *$ \\
\hline \multicolumn{5}{|l|}{ previous miscarriages } & \multirow{3}{*}{$0.020^{*}$} \\
\hline None & 210 & 77.8 & 231 & 85.6 & \\
\hline One or more & 60 & 22.2 & 39 & 14.4 & \\
\hline Mean \pm SD & \multicolumn{2}{|c|}{$0.27 \pm 0.56$} & \multicolumn{2}{|c|}{$0.19 \pm 0.54$} & 0.100 \\
\hline Menstrual cycle resumed & 122 & 45.2 & 249 & 92.2 & $0.000^{*}$ \\
\hline
\end{tabular}

$(P D B F)$ pregnancy during breastfeeding

(PAW) pregnancy after weaning

(*) Statistically significant difference 
Table (3): Effect of pregnancy during breastfeeding on the current pregnancy outcome.

\begin{tabular}{|c|c|c|c|c|c|}
\hline \multirow{2}{*}{ Items } & \multicolumn{2}{|c|}{$\operatorname{PDBF}(n=270)$} & \multicolumn{2}{|c|}{$\operatorname{PAW}(n=270)$} & \multirow{2}{*}{ P-value } \\
\hline & No. & $\%$ & No. & $\%$ & \\
\hline Lost follow up & 9 & 3.33 & 6 & 2.22 & 0.09 \\
\hline Complication during current pregnancy (\#) & 189 & 70.0 & 132 & 48.9 & $0.000 *$ \\
\hline Miscarriage & 6 & 2.2 & 1 & 0.4 & 0.284 \\
\hline Vaginal bleeding during pregnancy & 17 & 6.3 & 5 & 1.9 & 0.069 \\
\hline Placental separation & 18 & 6.7 & 5 & 1.9 & $0.050 *$ \\
\hline IUGR & 45 & 16.7 & 13 & 4.8 & $0.001 *$ \\
\hline Elevation of blood sugar & 16 & 5.9 & 15 & 5.6 & 0.387 \\
\hline Elevation of blood pressure & 33 & 12.2 & 42 & 15.6 & $0.003 *$ \\
\hline Eclampsia & 17 & 6.3 & 10 & 3.7 & 0.652 \\
\hline Anemia & 146 & 54.1 & 83 & 30.7 & $0.005 *$ \\
\hline
\end{tabular}

$(P D B F)$ pregnancy during breastfeeding

(*) Statistically significant difference

(PAW) pregnancy after weaning

(\#) Sum more than $100 \%$ as there was more than one answer.

Table (4): Delivery and its complication in the two study groups.

\begin{tabular}{|c|c|c|c|c|c|}
\hline \multirow{2}{*}{ Items } & \multicolumn{2}{|c|}{ PDBF $(n=270)$} & \multicolumn{2}{|c|}{ PAW $(n=270)$} & \multirow{2}{*}{ P-value } \\
\hline & No. & $\%$ & No. & $\%$ & \\
\hline Lost follow up & 9 & 3.33 & 6 & 2.22 & 0.09 \\
\hline \multicolumn{5}{|l|}{ Time of delivery } & \multirow{4}{*}{0.496} \\
\hline Full term delivery (At 37 weeks) & 185 & 68.5 & 179 & 66.3 & \\
\hline Preterm labor $(<37$ weeks $)$ & 42 & 15.6 & 53 & 19.6 & \\
\hline Post data $(>40$ weeks $)$ & 28 & 10.4 & 31 & 11.5 & \\
\hline \multicolumn{5}{|l|}{ Mode of delivery } & \multirow{4}{*}{$0.001 *$} \\
\hline Vaginal delivery & 137 & 50.7 & 178 & 65.9 & \\
\hline $\mathrm{CS}$ & 118 & 43.7 & 85 & 31.5 & \\
\hline \multicolumn{5}{|c|}{ Complications of normal delivery (\#) } & \\
\hline Ante-partum hemorrhage & 11 & 4.1 & 4 & 1.5 & $0.017 *$ \\
\hline Post-partum hemorrhage & 14 & 5.2 & 8 & 3.0 & $0.048 *$ \\
\hline Premature rupture of membranes & 29 & 10.7 & 34 & 12.6 & 0.649 \\
\hline Prolonged labor & 36 & 13.3 & 30 & 11.1 & $0.042 *$ \\
\hline
\end{tabular}

$(P D B F)$ pregnancy during breastfeeding

(*) Statistically significant difference

(PAW) pregnancy after weaning

(\#) Sum more than $100 \%$ as there was more than one answer.

Table (5): Newborn data in the two-study group.

\begin{tabular}{|c|c|c|c|c|c|}
\hline \multirow{2}{*}{ Items } & \multicolumn{2}{|c|}{ PDBF $(n=270)$} & \multicolumn{2}{|c|}{$\operatorname{PAW}(n=270)$} & \multirow{2}{*}{ P-value } \\
\hline & No. & $\%$ & No. & $\%$ & \\
\hline Lost follow up & 9 & 3.33 & 6 & 2.22 & 0.09 \\
\hline \multicolumn{5}{|c|}{ Condition of the newborn } & \multirow{2}{*}{0.118} \\
\hline Living birth & 249 & 92.2 & 262 & 97.0 & \\
\hline \multicolumn{5}{|l|}{ Birth weight } & \multirow{3}{*}{0.171} \\
\hline Mean \pm SD & \multicolumn{2}{|c|}{$2844.8 \pm 445.4$} & \multicolumn{2}{|c|}{$2897.0 \pm 414.1$} & \\
\hline Range & \multicolumn{2}{|c|}{$2000-4100$} & \multicolumn{2}{|c|}{$2000-4200$} & \\
\hline \multicolumn{5}{|l|}{ Birth weight } & \multirow{3}{*}{$0.017 *$} \\
\hline Normal & 210 & 77.8 & 239 & 88.5 & \\
\hline$<2500 \mathrm{gm}$ & 39 & 14.4 & 23 & 8.5 & \\
\hline
\end{tabular}




\begin{tabular}{|c|c|c|c|c|c|}
\hline \multirow{2}{*}{ Items } & \multicolumn{2}{|c|}{ PDBF $(n=270)$} & \multicolumn{2}{|c|}{ PAW $(n=270)$} & \multirow{2}{*}{ P-value } \\
\hline & No. & $\%$ & No. & $\%$ & \\
\hline \multicolumn{5}{|c|}{ Time of starting breast feeding after delivery } & \multirow{4}{*}{0.782} \\
\hline Within 1 hour & 88 & 32.6 & 94 & 34.8 & \\
\hline Within 6 hours & 135 & 50.0 & 136 & 50.4 & \\
\hline More than 6 hours & 26 & 9.6 & 32 & 11.9 & \\
\hline Referred to PICU & 117 & 43.3 & 106 & 39.3 & 0.137 \\
\hline
\end{tabular}

$(P D B F)$ pregnancy during breastfeeding

(PICU) pediatric intensive care unite

Table (1) : the mean age of the women was $28.82 \pm$ 6.18 in the PDBF group and $30.52 \pm 5.20$ in the PAW group with statistically significant difference $(\mathrm{P}=0.001)$. The percentage of women's husbands who were working abroad was significantly higher in women who got pregnant during breastfeeding compared with women who got pregnant after weaning (13.0 \% and $7.4 \%$, respectively). The remaining data presented in table 1

Table (2) : the obstetric history of the studied women is presented in table 2. Almost, more than half of the PDBF group $(61.5 \%)$ and about three fourth of the PAW group $(84.4 \%)$ had more than one previous deliveries with statistically significant difference, with a mean value in the PDBF group was $(2.39 \pm$ $1.54)$ and in the PAW group $(2.64 \pm 1.18),(\mathrm{P}=0.036)$. Concerning the history of previous miscarriages, the majority of women in both groups, $(77.8 \%)$ in the PDBF group and $(85.6 \%)$ in the PAW group, had no previous miscarriages, with no statistically significant difference $(\mathrm{P}=0.100)$.

Table (3) : regarding the effect of pregnancy-location overlap on the pregnancy outcome the percentage was higher in the PDBF group $(70.0 \%)$ compared with $(48.9 \%)$ in PAW group with statistical significant difference $(\mathrm{P}=0.000)$. The miscarriage percentage was higher in the PDBF group (2.2\%) compared to $(0.4 \%)$ in the PAW group, However, the difference observed were not statistically significant $(\mathrm{P}=0.284)$. Anima had the highest percentage in both PDBF group and PAW group $(54.1 \%$ and $30.7 \%$, respectively), $(\mathrm{P}=0.005)$.As regard, the percentage of IUGR was higher in the PDBF group (16.7\%) compared with (4.8\%) in the PAW group. However, elevation of blood pressure was higher in the PAW group (15.6\%) compared with (12.2\%) in the PDBF group with statistical significant difference, $(\mathrm{P}=0.003)$.

Table (4) : demonstrates that the percentage of women who had cesarean section was higher in the PDBF group (43.7\%) compared with women in the PAW group $(31.5 \%),(\mathrm{P}=0.001)$. More than half of women in the PDBF group (82.6\%) had complication during normal delivery compared with $(62.2 \%)$ had complication in the PAW group, $(\mathrm{P}=0.000)$.
(PAW) pregnancy after weaning

(*) statistically significant difference
Prolonged labor was higher percentage in the PDBF group $(13.3 \%)$ compared with $(11.1 \%)$ in the PAW group $(\mathrm{P}=0.042)$. As regarded, post-partum hemorrhage was higher in the PDBF group $(5.2 \%)$ compared with $(3.0 \%)$ in the PAW group and the difference was statistically significant $(\mathrm{P}=0.048)$, Women in the PDBF group had higher percentage in Ante-partum hemorrhage compared with woman in the PAW group (4.1\% and $1.5 \%$ respectively), and the difference was statistically significant $(\mathrm{P}=0.017)$.

Table (5) : showed the newborn data in the two groups. The mean birth weight of the study sample was $(2844.8 \pm 445.4)$, in the PDBF group and $(2897.0 \pm 414.1)$ in the PAW group $(\mathrm{P}=0.171)$, The percentage of low birth weight was significantly higher in the PDBF group (14.4\%) compared with $(8.5 \%)$ in the PAW group $(\mathrm{P}=0.017)$. The percentage of newborn referral to PCU was higher in the PDBF group $(43.3 \%)$ compared with $(39.3 \%)$ in the PAW group. However, no statistically significant difference was observed $(\mathrm{P}=0.137)$.

\section{Discussion}

Women in Egypt and many other developing countries commonly breastfeed their children for long periods of time, usually for two years (Speroff and Mishell, 2008; WHO, 2004). That is why, a good number of women in these societies get pregnant during breastfeeding (Shaaban and Glasier, 2008). These women usually have high total fertility rate and almost inadequate dietary intake with short interpregnancy interval. Therefore, when lactation overlaps with pregnancy in these women, large burden is added to their nutritional status (Sengul et al., 2012). The current study is trying to Study the effect of pregnancy during lactation on the maternal and prenatal outcome and to study the effect of duration of lactation and time of weaning on the pregnancy outcome.

The socio-demographic characteristic of the PDBF group and the PAW group were closely similar, with a large proportion of educated, not working and Muslim. Husbands of women in PDPF group are more commonly working outside the wife's residence 
area (either in other city or abroad) compared with husbands of women in PAW group. Female partners, whose husbands not living with them, are usually reluctant about using a long term method of contraception as sexual intercourse is much infrequent, especially during lactation as they feel extra security from the contraceptive effect of breastfeeding. This is in addition to traditional believe among women who's husbands are traveling, that the use of contraception is a sin and a kind of accuse in their marital unfaithfulness.

Women who got pregnant after weaning is significantly older than those got pregnant during breast-feeding. Women in PAW group had had longer duration of marriages and higher parity and greater number of living children. They were more caring about the use of contraceptive methods. This may not be the case in PDBF group who was usually of younger age and parity with probably not yet completed their family life. Our findings, contradicted studies in our setting which compared contraceptive use among Two hundred and thirtythree parous Egyptian women with unplanned pregnancies less than 2 years after delivery, were comparing between PDBF group and PAW group and reported that there was no significant difference between the ages of the two groups (Tilley et al., 2009).

The present results disagreed with the study done in Turkey included 61 pregnant women who had inter pregnancy intervals of two years were evaluated. To assess the outcomes of the pregnancies of lactating women who found no significant difference between the mean ages in the two study groups (Sengul et al., 2012). The difference may be secondary to the difference in the nature of the community involved in the different studies. The current study is conforming to previous study which studied 133 Peruvian pregnant women, 68 breastfed during the last trimester of pregnancy, and 65 had not breastfed during pregnancy and finding no difference between the level of education in women who got pregnant during breast-feeding and after weaning (Marquis et al., 2002).

On the other hand it disagrees with the study by Tilley and colleagues who found that there was a significantly greater percentage of literate women in the PAW group than in the PDBF group (Tilley et al., 2009) and assumed that, the more the education of the mother the more the awareness of necessity of women to use contraception after stoppage of lactation.

Concerning with the history of previous miscarriages, the present study revealed higher percentage of women with history of one or more miscarriage in PDBF group (22.2\%) compared with PAW group
(14.4\%). Simply this may be secondary of having younger women in PDBF group and of less experience of self-care during pregnancy. The above results agreed with (Sengul et al., 2012) who found significant increase in abortion rate in PDBF group compared with PAW group $(0.79 \pm 0.86$ and $0.05 \pm$ $0.21)$ respectively $(\mathrm{p}=0.01)$.

The present work revealed that $45.2 \%$ of women in PDBF group had resumed their menses before the current pregnancy. These results are consistent with the study in the same setting for 2617 parous women attending a hospital in Egypt for antenatal care by Shaaban and Glassier, 2008 that demonstrated the menstrual cycle resumed in about $84.9 \%$ in this group of women, which get pregnant during breastfeeding.

Regarding the duration of lactation -pregnancy overlap, our study showed that more than half of the women breast-fed their children for the first four months of the current pregnancy. Women usually wait until they become completely sure about the integrity of their current pregnancy to stop breastfeeding. Stoppage of lactation during pregnancy is partially natural as the amount and the quality of breast milk decrease with pregnancy and partially secondary to concerns about maternal health "already malnourished" that is sometimes advised by treating doctors. (Merchant et al., 1990) who conducted a study among rural Guatemalan women participating in a nutrition supplementation trial, lactation overlapped with pregnancy in 253 of 504 $(50.2 \%)$ of the pregnancies, and found that $55.7 \%$ of women breast-fed only during the first three months of pregnancy.

The present work disclosed a statistically significant higher incidence of having more pregnancy complication (collectively) in women got pregnant during breastfeeding compared with those got pregnant after weaning $(70.0 \%$ and $48.9 \%$, respectively).The above results contradicting the results of Madarshahian, (2012) who conducted a study over 9 months, which examined two groups of women in the maternity units of two hospitals in Birjand, Iran. The first group comprised 80 women who breast-fed for 30 days or more during pregnancy; the second group comprised 240 women who did not. Who found no statistical significant difference in the problem during pregnancy between the overlap group and the non overlap group. Moreover, the present finding contradicted what is demonstrated by Sengul et al., (2012). The difference between our study and the above two studies could be basically the difference on the basal nutritional point from where women in the different study started their pregnancies. 
Miscarriage rate, although it is our primary outcome, there was no statistically significant difference observed between both groups with regard miscarriage. These results agreed with the results of (Madarshahian et al., 2012). On the other hand, these results disagreed with (Albadran, 2013) who conducted a study in 340 pregnant women in Iraqi and found that there was significantly higher frequency of miscarriage among those who got pregnant after weaning $(10.35 \%)$ compared with $(5.12 \%)$ of those who breastfed during pregnancy ( $\mathrm{P}$ $<0.0164)$.

The results of present study revealed significant higher occurrence of hypertensive disorders with pregnancy in PAW group as compared with PDBF group $\left(p=0.003^{*}\right)$ this result disagreed with (Madarshahian et al., 2012) who found that there was no relation between overlap and non overlap groups with hypertension, this finding may be explained by low dietary intake of calcium in our community .This result agreed with a study mad by Murphy et al., 1992 , on 255 toddlers aged 18-30 ,who found that calcium intakes were, well, below recommended amounts in his study which estimated mineral intakes of toddlers; in Egypt, Kenya, and Mexico.

The results of present study showed that women in PDBF suffered from more delayed fetal growth, $(\mathrm{P}=0.001)$ as compared with women in PAW group. This result is in the same line with results of (Merchant et al., 1990) that suggested that the overlap could produce suboptimal outcomes for both pregnancy and subsequent lactation, such as IUGR of the fetus. Moreover, this study agreed with (Van Eijsden et al., 2008), who suggested that folate depletion contributes to the risk of IUGR, which is associated with short interpregnancy intervals. However, these results disagreed with (Sengul et al., 2012) who reported that there was no difference in IUGR between the two groups. The basic nutritional status of women in our culture is much different from others.

Moreover, more than half of pregnant women during breastfeeding $(54.1 \%)$ were suffering from anemia, a factor that is probably contributed. This finding agreed with (Dairo and Lawoyin 2004) who found an association between interpregnancy intervals shorter than 24 months and increased risk of anemia during pregnancy. Short intervals could indirectly increase the risk of adverse neonatal/infant outcomes through changes in breastfeeding patterns or the composition and/or quantity of breast milk secondary to breastfeeding-pregnancy overlap.

According to the findings of the current study, it can be observed that women who got pregnant during breastfeeding had higher incidence of cesarean delivery $(43.7 \%)$ as compared with women who got pregnant after weaning $(31.5 \%)(p=0.001 *)$. The difference may be secondary that this pregnancy may be more risky. Moreover may be explained by having shorter inter-pregnancy interval in women who got pregnant during breastfeeding usually gave no enough time for adequate healing of CS scar, If they had delivered by CS in previous pregnancy.

The present study revealed that there was increase incidence of having more prolonged labor in women who got pregnant during breastfeeding as compared with those who got pregnant after weaning. These results agreed with (Marquis et al., 2002) who found that PDBF mothers tended to have a higher incidence of prolonged labor $(\mathrm{P}=0.042)$ than the PAW mothers. On the other hand, this result disagreed with (Pareja, 2007) who found that there no differences were found in the proportion of mothers who breastfed during late pregnancy between the cases of prolonged active Phase of labor (PAPL) and their respective controls.

The present work showed more significant incidence of low birth weight in women who got pregnant during breastfeeding. This result was in the same line with (Sengul et al., 2012) that found a significant decrease in birth weight observed in these women who became pregnant during lactation. The lower birth weight of infants in the overlap group may be because of anemia and lack of maternal milk consumption during pregnancy. However, this result disagreed with (Madarshahian, 2012 \& Albadran, 2013) that showed no difference in neonatal birth weight between PDBF and PAW groups.

The results of present study revealed that there was no significant relation between the duration of lactation-pregnancy overlap and the current pregnancy and complications, This result was in the same line with (Sengul et al., 2012) who showed that the duration of lactation during the subsequent pregnancy period is not a determining factor in whether pregnancy of lactating woman is associated with complications or not.

\section{Limitation of the study}

- Transportation was a problem especially when go to the maternal and child health centers $(\mathrm{MCH})$ in Assiut governorate (Kolta $\mathrm{MCH}$, Hay ktharb $\mathrm{MCH}$, Elarbaeen $\mathrm{MCH}$, Hay shark $\mathrm{MCH}$ and Manfalote MCH).

- Few of the studied samples refused to participate in the study.

- The study did not cover the entire communities in the governorate due to lack of resources such as financial support, time and personnel. 


\section{Conclusion}

Pregnancy during breast-feeding is common in societies with high total fertility rate and it is commonly unintended. PDBF dose not carry higher incidence of miscarriage rate when compared with PAW, but it carries higher incidence of placental abruption, delayed fetal growth and anemia of pregnancy, antepartum and postpartum hemorrhage, premature rupture of membranes and prolonged labor together with more incidence of having cesarean delivery.

\section{Recommendations}

- An education program should be implemented for mothers and different health personnel working in $\mathrm{MCH}$ centers about the risk of a possible adverse prenatal outcome.

- Since the continuation of the lactation does not deteriorate the pregnancy outcome, however, breastfeeding should not be immediately stopped while taking into consideration the nutritional status of the mother.

- Since Pregnancy during breastfeeding is common in Egypt, Further studies are needed to validate our results and support our study.

\section{Reference}

1. Dairo, Magbagbeola D., and Taiwo $O$., Lawoyin, (2004): "Socio-demo-graphic determinants of anemia in pregnancy at primary care level: A study in urban and rural Oyo State, Nigeria," African Journal of Medicine and Medical Sciences; 33(3): 213-217.

2. Fathalla M., Abdel-Raheem M., and Amin A. (2003): The prevalence, determinants and outcome of unintended pregnancy: a hospitalbased study. J Egypt Soc Obstet Gynecol; 29: 945-54.

3. Khella A., Fahim H., Issa A., Sokal D., and Gadalla MA, (2004): Lactational amenorrhea as a method of family planning in Egypt. Contraception, 69:317-22.

4. Madarshahian F., and Hassanabadi M., (2012): A comparative study of breastfeeding during pregnancy: impact on maternal and newborn outcomes. J Nurs Res.; 20(1): 74-80.

5. Marquis G., Penny M., Diaz J., Judith M., Diaz and R., Marín M., (2002): Postpartum Consequences of an Overlap of Breastfeeding and Pregnancy Reduced Breast Milk Intake and
Growth during Early Infancy, PEDIATRICS . 109 (4).1-8.

6.

Maysara M., and Albadran, (2013): Effect of Breastfeeding during Pregnancy on the Occurrence of Miscarriage and Preterm Labour. Iraqi Journal of Medical Sciences; 11(3):285-89.

7. Merchant K., Martorell R., and Haase J., (1990): Maternal and fetal responses to the stresses of lactation concurrent with pregnancy and of short recuperative intervals. Am J Clin Nutr. 3(52):280-288.

8. Ministry of health, (2006): food and nutrition guidelines for healthy, pregnant and breastfeeding women; 2-3.

9. Murphy S., Beaton G., and Calloway D., (1992): Estimated mineral intakes of toddlers: predicted prevalence of inadequacy in village populations in Egypt, Kenya, and México; Am J Clin Nutr 56 ( 3).565-572.

10. Pareja R., (2007): The association between breastfeeding during late pregnancy and the occurrence of small for gestational age and prolonged active phase of labor among Peruvian women 21(4) :1-14.

11. Sengul O., Sivaslioglui A., Kokanali M., and Ustuner I., (2012): The outcomes of the pregnancies of lactating women, Turkish Journal of Medical Sciences 43: 251-254.

12. Shaaban O., and Glasier A., (2008): Pregnancy during breastfeeding in rural Egypt. Contraception, 77:350-354.

13. Smith G., Pell J., and Dobbie R., (2003): Interpregnancy interval and risk of preterm birth and neonatal death: retrospective cohort study. BMJ; 313- 327.

14. Speroff L and Mishell DR, (2008): The postpartum visit: it's time for a change in order to optimally initiate contraception. Contraception, 78: 8-90.

15. Tilley B., Shaaban M., Wilson M., Glasier A., Daniel R., and Mishell J., (2009): Breastfeeding and contraception use among women with unplanned pregnancies less Than 2 years after delivery. International Journal of Gynecology and Obstetrics International Journal of Gynecology and Obstetrics 105: 127-130.

16. Van Eijsden M., Smits L., Van der Wal M., and Bonsel G., ( 2008): Association between short interpregnancy intervals and term birth weight, the role of folate depletion. Am J Clin Nutr; 88: 147-53. 